\title{
INFLUENCIA DEL FRÍO INVERNAL Y PORTAINJERTOS EN LA BROTACIÓN Y FLORACIÓN DEL MANZANO ROYAL GALA
}

\section{CHILLING WINTER AND ROOTSTOCKS INFLUENCE ON ROYAL GALA APPLE BUD BREAKING AND BLOOMING}

\author{
Izdiana M. Peña-Antillón ${ }^{1}$, Juan L. Jacobo-Cuéllar ${ }^{1 *}$, Rafael A. Parra-Quezada ${ }^{1}$, \\ Víctor M. Guerrero-Prieto', Manuel R. Ramírez-Legarreta ${ }^{2}$, Dámaris L. Ojeda-Barrios ${ }^{1}$, \\ Ofelia A. Hernández-Rodríguez ${ }^{1}$ y Nora A. Salas-Salazar ${ }^{1}$
}

\begin{abstract}
'Universidad Autónoma de Chihuahua, Facultad de Ciencias Agrotecnológicas, Ciudad Cuauhtémoc, Chihuahua, México. ${ }^{2}$ Instituto Nacional de Investigaciones Forestales, Agrícolas y Pecuarias, Campo Experimental Sierra de Chihuahua (Ex-investigador), Ciudad Cuauhtémoc, Chihuahua, México.
\end{abstract}

*Autor de correspondencia (iljacobo@uach.mx)

\section{RESUMEN}

El manzano (Malus sylvestris var. domestica) se cultiva en gran diversidad de ambientes, con diferentes portainjertos y cultivares, por lo que la caracterización de la respuesta de la planta en cada una de las combinaciones ambiente-cultivar-portainjerto es importante para entender el comportamiento del árbol en su entorno. Con el objetivo de explorar la influencia del frío invernal expresado en unidades frío (UF) acumuladas durante ese periodo y de portainjertos sobre la apertura de yemas y floración del manzano, se seleccionó un huerto con la variedad Royal Gala injertada sobre 12 portainjertos diferentes. En los inviernos 2010-2011 y 2014-2015 se calcularon las unidades frío y posterior a ésto, el porcentaje de yemas abiertas y los grados día (GD) requeridos para el inicio (5\%) y durante el evento de floración (de 5 a $95 \%$ ) para cada una de las combinaciones cultivar/ portainjerto. La apertura de yemas y el evento de floración de Royal Gala se midieron periódicamente en porcentaje relativo de ocurrencia y se relacionó con UF y portainjerto. Posterior a un invierno con baja acumulación de frío (524 UF) abrió el $72.6 \%$ de yemas, la floración inició a los 236 GD y se completó en $117 \mathrm{GD}$, mientras que posterior a un invierno con 830 UF abrió el $96.7 \%$ de yemas, la floración inició a los 146 GD y se completó en 129 GD. Se detectó influencia significativa del factor frío invernal para la apertura de yemas y para los GD acumulados al inicio de la floración e igualdad estadística para la duración de la floración. No se detectó efecto significativo de portainjerto ni interacción significativa entre frío invernal y portainjerto.

Palabras clave: Malus sylvestris var. domestica, floración, días grado, portainjertos, unidades frío.

\section{SUMMARY}

The apple tree (Malus sylvestris var. domestica) is grown in a wide variety of environments, with different rootstocks and cultivars; thus, the characterization of the response of the plant in each of the environmentcultivar-rootstock combinations is important to understand the tree behavior in its environment. In order to explore the influence ofwinter cold expressed in chill units (CU) accumulated during the winter period as well as rootstocks on bud breaking and blooming of apple trees, an apple orchard with the Royal Gala cultivar grafted on 12 different rootstocks was selected. During the winter periods of 2010-2011 and 2014-2015 CU were calculated and after that, the percentage of open buds and the day degrees (DD) required for the beggining (5\%) and during the flowering event (from 5 to $95 \%$ ) for each of the environment-cultivar-rootstock combination were registered. The bud opening and blooming of Royal Gala were periodically measured in percentage of occurrence and related to CU, DD and rootstock. After a winter with low chill units accumulated (524 CU) there was $72.6 \%$ of bud breaking, blooming began at $236 \mathrm{DD}$ and was completed with $117 \mathrm{GDD}$, while after a winter with $830 \mathrm{CU}$ there was $96.7 \%$ of bud breaking, blooming began at $146 \mathrm{DD}$ and was completed with 129 DD. Significant difference for winter cold factor was detected on bud breaking and DD accumulated at the beginning of blooming and statistical equality for the duration of blooming period. No significant effect of rootstocks or significant interaction between CU and rootstocks were detected.

Index words: Malus sylvestris var. domestica, blooming, degree days, rootstocks, chill units.

\section{INTRODUCCIÓN}

El manzano (Malus sylvestris var. domestica) se cultiva en gran diversidad de ambientes, con diferentes portainjertos y cultivares, por lo que la caracterización de la respuesta de la planta en cada una de las combinaciones ambientecultivar-portainjerto es un principio básico para entender el comportamiento del árbol en su entorno. En el oeste del estado de Chihuahua, como en otras áreas, el sistema de producción de manzano es afectado por variaciones intra e interanuales en temperaturas máximas, mínimas y precipitación (Ramírez et al., 2011). En un mismo invierno se encuentran diferencias hasta 60 \% en la acumulación de unidades frío con el método propuesto por Richardson et al. (1974) cuando se comparan sitios contiguos (e.g. $28^{\circ}$ $13^{\prime} \mathrm{N}, 106^{\circ} 83^{\prime} \mathrm{O}, 1894$ msnm; $29^{\circ} 18^{\prime} \mathrm{N}, 107^{\circ}$ 50' O, 2144 msnm).

El portainjerto es un componente básico en huertos de manzano y tiene influencia en el vigor del árbol, precocidad, eficiencia de producción, absorción mineral, resistencia a condiciones ambientales adversas y tamaño del fruto (Malagi et al., 2015; Marini et al., 2014). 
En relación con el efecto del portainjerto sobre floración en cultivares de manzano puede destacarse el estudio de Tabuenca y Gracia (1971), quienes evaluaron 18 combinaciones generadas de tres portainjertos y seis variedades y reportaron diferencias sólo para el inicio de brotación, mientras que, en duraznero [Prunus persica (L.) Batsch], Durner y Goffreda (1992) encontraron efecto variable de portainjertos en brotación y tasa de desarrollo de yemas en algunos cultivares.

Los árboles de clima templado, como el manzano, están sujetos a una etapa de letargo en la que reducen su actividad fisiológica a un mínimo (Atkinson et al., 2013) y para superarla debe satisfacerse un requerimiento de frío, específico para cada especie y cultivar (Samish, 1954) y modificada por las condiciones ambientales imperantes (Allen y Wann, 1983). La reducida acumulación de frío invernal y la ocurrencia de temperaturas cálidas al final del reposo han fundamentado la variabilidad en el evento de floración en diferentes áreas (Legave et al., 2013). En manzano, la baja acumulación de frío propicia reducción en la brotación y en el crecimiento vegetativo, así como retraso y alargamiento del periodo floral (Atkinson et al., 2013; Legave et al., 2015).

Al final del invierno y previo a la brotación de yemas debe acumularse calor para iniciar y completar el desarrollo de la yema (Luedeling, 2012); de hecho, la dinámica de crecimiento de frutales templados es resultado de mecanismos fisiológicos complejos y de su interacción con el ambiente (Malagi et al., 2015), del cual se ha distinguido a la temperatura como uno de los indicadores climáticos con mayor influencia en el desarrollo de yemas florales (Luedeling, 2012).

La respuesta del manzano en climas contrastantes es lo apropiado para la caracterización del reposo y la dinámica de crecimiento en sitios con reducida acumulación de frío, condiciones que se presentarán en algunas áreas actualmente identificadas como de clima templado (Malagi et al., 2015); en este caso particular, se provee de información de dos años, en una misma zona semiárida (García, 2004), donde se cultiva manzano y a partir del invierno 2004-2005 se detectaron cambios significativos en la acumulación de unidades frío (Ramírez et al., 2011).

Con los antecedentes citados, el objetivo de este trabajo fue determinar la influencia del frío invernal y portainjertos en la brotación de yemas e inicio y duración del evento de floración en manzano Royal Gala.

\section{MATERIALES Y MÉTODOS}

\section{Sitio experimental}

El trabajo se realizó durante los años 2010-2011 y 20142015 en un huerto de 10 años de plantado (al año 2010) en el municipio de Cuauhtémoc, Chihuahua, México ubicado a $28^{\circ} 25^{\prime} 55^{\prime \prime} \mathrm{N}$ y $106^{\circ} 52^{\prime} 42^{\prime \prime} \mathrm{O}$, altitud de 2033 msnm, con clima seco templado y verano cálido, temperatura media anual entre 12 y $18^{\circ} \mathrm{C}$ con oscilaciones muy extremosas, lluvias en verano y con menos del $5 \%$ de lluvia invernal (García, 2004).

\section{Material vegetal}

Se utilizó manzano Royal Gala injertado sobre los portainjertos clonales B.9, G.11, G.16, G.30, G.41, G.935, G.4210, G.6210, M.7, M.26, M.9PAJAM2 y M.9T337, con alto requerimiento de frío, de acuerdo con su origen (Denardi et al., 2015). Al momento de la plantación (10 años antes) cada uno de los árboles se estableció con asignación aleatoria en dos hileras, con 10 repeticiones por combinación Royal Gala/portainjerto, 1.5 m entre árboles y 4.5 m entre hileras.

\section{Condiciones meteorológicas}

Se obtuvo información de temperaturas máximas, mínimas y precipitación pluvial $(\mathrm{mm})$ durante los periodos de septiembre del año 2010 a mayo del año 2011 y de septiembre del año 2014 a mayo del año 2015 del sitio denominado Quintas Lupita, que cuenta con una estación agroclimática (Campbell Scientific ${ }^{\mathrm{MR}}$ automatizada, Logan, Utah, EUA) con data logger CR 10X, con sensores para variables climáticas y a 1600 m de distancia del sitio experimental.

Se analizó la ocurrencia de temperaturas máximas y mínimas, su intervalo, y se calcularon unidades frío (UF) con el procedimiento propuesto por Richardson et al. (1974) durante los inviernos 2010-2011 y 2014-2015, con inicio a partir de una acumulación consistente de UF y con término el 28 de febrero para ambos inviernos; con base en lo anterior, se definieron dos niveles de frío invernal, uno con baja acumulación de frío (524 UF) ocurrida durante el invierno 2010-2011 y otro con alta acumulación de frío (830 UF) ocurrida durante el invierno 2014-2015. Se analizó también la precipitación pluvial $(\mathrm{mm})$ ocurrida durante los inviernos considerados. 


\section{Brotación y cinética de floración de Royal Gala}

Durante los ciclos 2011 y 2015 se ubicaron árboles de manzano Royal Gala injertados sobre los portainjertos mencionados; dentro de cada combinación, los árboles se homogenizaron por área de la sección transversal del tronco y se eligieron tres árboles de manera aleatoria, en cada uno se marcaron cuatro ramas con orientación cardinal, en cada una de las ramas se ubicaron los crecimientos de dos y tres años y se contó el número de yemas en los dos crecimientos en cada una de las ramas por árbol. Se obtuvo el porcentaje de brotación relativa con las yemas totales y yemas abiertas (florales y vegetativas). Posteriormente, en estos mismos crecimientos, ramas y árboles por combinación Royal Gala/portainjerto y frío invernal, en dos ocasiones por semana se contó el número de racimos florales, se acumularon por fecha y se obtuvo la frecuencia relativa por fecha de lectura por árbol desde el inicio hasta el término de la floración.

A partir del 1 de marzo, y hasta que se completó el evento de floración, se calcularon grados día (GD) con temperatura base de $4.5^{\circ} \mathrm{C}$ (Whiting et al., 2015), seno simple y corte horizontal, en la plataforma interactiva de UCANR (2019). Los GD se consideraron como variable independiente y se relacionaron con la cinética de floración por árbol con el modelo no lineal de Weibull modificado (Pennypacker et al., 1980) en la versión integrada de la forma:

$$
y=7-e^{\left[-(t / b)^{c}\right]}
$$

donde y en fue la proporción de floración por unidad de tiempo, $t$ tiempo en grados día (GD), $1 / b$ es un estimador de la tasa de incremento del evento de floración por unidad de tiempo (GD) y c es un parámetro de la forma de la curva y carece de unidades.

En total se obtuvieron 72 modelos producto de un modelo por árbol (tres repeticiones, 12 portainjertos y dos periodos de evaluación). Cada modelo permitió estimar el requerimiento de GD para inicio y duración del periodo de floración. El modelo Weibull en la versión simplificada se ha aplicado en diferentes situaciones con ajustes significativos y coeficientes de determinación superiores al 93 \% (Jacobo-Cuellar et al., 2005; Mora-Aguilera et al., 1996). La modelación se realizó en el sistema de análisis estadístico SAS ver. 9.3 (SAS Institute, 2011).

\section{Análisis estadístico y arreglo experimental}

Las temperaturas máximas y mínimas ocurridas durante los inviernos se compararon con intervalos del $95 \%$ de confianza para las medias, con el criterio de decisión: igualdad estadística si se comparten valores dentro de los intervalos de confianza $\left(\mathrm{IC}_{95 \%}\right)$ (Sincich et al., 2002). El requerimiento de GD para brotación, inicio (5\%) y duración del evento de floración (5-95 \%) se analizó bajo un arreglo de tratamientos de tipo factorial, con unidades frío y portainjerto como factores, alta (830) y baja (524) acumulación de UF y doce portainjertos como niveles, respectivamente, bajo un diseño experimental completamente aleatorio. Efectos significativos de factores o interacción entre niveles en el análisis de varianza fueron separados por comparación múltiple de medias con la prueba de Tukey $(P \leq 0.05)$.

\section{RESULTADOS}

\section{Temperaturas invernales y acumulación de grados día (GD)}

Con base en los $\mathrm{IC}_{95 \%}$ los promedios de las temperaturas mínimas registradas durante los dos inviernos evaluados fueron estadísticamente iguales entre sí, con valores de -1.6 $\pm-0.8^{\circ} \mathrm{C}$ y de $-0.1 \pm 0.7^{\circ} \mathrm{C}$ para los inviernos $2010-2011$ y 2014-2015, respectivamente, mientras que los promedios para las temperaturas máximas fueron estadísticamente diferentes con valores de $18.4 \pm 0.8^{\circ} \mathrm{C}$ y $16.8 \pm 0.7^{\circ} \mathrm{C}$ para los invierno 2010-2011 y 2014-2015, respectivamente. En el invierno de 2010-2011 no se registró precipitación, mientras que en el de 2014-2015 la precipitación fue de $46 \mathrm{~mm}$; el 70 \% de la precipitación se distribuyó en seis eventos con precipitaciones $\geq 3 \mathrm{~mm}$.

Posterior a los dos periodos de frío, con inicio el 1 de marzo y hasta que se completó el evento de floración, se acumularon 606 GD para el año 2011 y 467 GD para el año 2015.

\section{Apertura de yemas, inicio y duración del evento de floración}

La apertura de yemas de manzano Royal Gala difirió estadísticamente sólo para acumulación de frío. En el invierno con baja acumulación de frío (520 UF) abrió el 72.6 $\%$ de las yemas consideradas, mientras que en el invierno con alta acumulación de frío (830 UF) abrió el $96.8 \%$ (Cuadro 1). Con relación al factor portainjerto, el porcentaje de yemas abiertas de Royal Gala fue estadísticamente igual sobre los diferentes portainjertos. La menor apertura de yemas ocurrió cuando estuvo sobre el portainjerto G935 con $73.2 \%$, mientras que la mayor apertura de yemas se detectó sobre el portainjerto M9 con 98.6 \% (Cuadro 1). No se detectó interacción significativa entre los niveles de frío y portainjertos. 
Cuadro 1. Frecuencia relativa media (\% \pm error estándar) de yemas abiertas por árbol de manzano Royal Gala sobre diferentes portainjertos y frío invernal (UF) en Cuauhtémoc, Chihuahua, México.

\begin{tabular}{|c|c|c|c|c|c|}
\hline \multirow{2}{*}{ Portainjerto } & \multicolumn{4}{|c|}{ Frío invernal (Unidades Frío) } & \multirow{2}{*}{$\begin{array}{l}\text { Frecuencia relativa (\%) } \\
\text { Royal Gala/portainjerto }\end{array}$} \\
\hline & $\mathrm{n}$ & Bajo (524) & $\mathrm{n}$ & Alto (830) & \\
\hline B.9 & 52 & 71.5 & 84 & 89.6 & $80.5 \pm 6.2 \mathrm{a}$ \\
\hline G.11 & 58 & 74.0 & 70 & 97.0 & $85.7 \pm 6.4 \mathrm{a}$ \\
\hline G.16 & 74 & 61.7 & 86 & 99.6 & $80.6 \pm 8.7 \mathrm{a}$ \\
\hline G.30 & 49 & 69.6 & 66 & 99.5 & $84.5 \pm 8.2 \mathrm{a}$ \\
\hline G.41 & 75 & 74.7 & 60 & 97.6 & $86.2 \pm 5.3 a$ \\
\hline G.4210 & 54 & 81.1 & 70 & 95.3 & $88.2 \pm 3.7 \mathrm{a}$ \\
\hline G.6210 & 71 & 75.0 & 67 & 99.5 & $87.3 \pm 5.8 a$ \\
\hline G.935 & 77 & 48.7 & 60 & 97.6 & $73.2 \pm 12.8 \mathrm{a}$ \\
\hline M.26 & 70 & 83.7 & 77 & 92.2 & $87.9 \pm 3.8 \mathrm{a}$ \\
\hline M.7 & 55 & 71.4 & 58 & 98.6 & $98.6 \pm 6.5 a$ \\
\hline M.9PAJAM2 & 48 & 80.9 & 67 & 95.1 & $88.0 \pm 3.4 \mathrm{a}$ \\
\hline М.9Т337 & 73 & 78.8 & 43 & 98.6 & $88.7 \pm 6.1 \mathrm{a}$ \\
\hline $\begin{array}{l}\text { Frecuencia } \\
\text { relativa } \\
\text { por frío invernal }\end{array}$ & \multicolumn{2}{|c|}{$72.6 \pm 2.4 \mathrm{a}$} & \multicolumn{2}{|c|}{$96.7 \pm 0.8 b$} & \\
\hline
\end{tabular}

n: número de yemas en crecimientos del segundo y tercer año en cuatro ramas por árbol. Valores con misma letra en hilera frecuencia relativa por frío invernal significa igualdad estadística entre ellos, mientras que valores con misma letra en columna de la derecha significa igualdad estadística entre portainjertos (Tukey, $\mathrm{P} \leq 0.05$ ).

El modelo Weibull modificado (Pennypacker et al., 1980) ajustó de manera significativa cada uno de los eventos de floración, obteniéndose coeficientes de determinación superiores al $90 \%$ y coeficientes de variación menores al $22 \%$. Con cada una de las ecuaciones generadas se estimó el requerimiento de GD para el inicio (5\%) y duración del periodo de floración (diferencia de GD entre 5 y $95 \%$ ). Para el inicio de floración se detectó diferencia significativa para el factor frío invernal, ya que posterior a una baja acumulación de frío (524 UF) Royal Gala requirió en promedio de 236 GD para iniciar la floración, mientras que posterior a una acumulación alta de frío (830 UF) el evento de floración inició a los 117 GD (Cuadro 2).

Con respecto a portainjertos, el inicio de floración de Royal Gala ocurrió entre 168 GD sobre M.9PAJAM2 y 219 GD sobre G.41, con igualdad estadística entre sí (Cuadro 2). No se detectó interacción significativa entre los niveles de frío y portainjertos.

La duración de la floración de Royal Gala fue estadísticamente igual para frío invernal y portainjertos y no se detectó interacción significativa entre niveles de frío y portainjertos. Posterior a un invierno con baja acumulación de frío (524 UF), Royal Gala requirió de 117 GD para completar la floración, mientras que después de un invierno con alta acumulación de frío (830 UF) lo completó en 129 GD (Cuadro 2). Con respecto a portainjertos, Royal Gala completó el evento de floración entre 100 GD sobre G.41 y 144 GD sobre M.7 sin diferencias estadísticas entre ellos (Cuadro 2).

\section{DISCUSIÓN}

En publicaciones referidas al clima y su relación con el desarrollo floral en frutales se utilizan diferentes indicadores climáticos y profundidad en su descripción, por lo que el contraste de resultados entre latitudes es limitado. A la descripción del clima que impera en el oeste de Chihuahua (García, 2004), y con base en los resultados observados deberá agregarse una oscilación media de 21.6 ${ }^{\circ} \mathrm{C}$ en temperaturas diarias invernales y precipitaciones que pueden variar desde 0 hasta $46 \mathrm{~mm}$. Esta condición inestable en la temperatura y precipitación se suma a un incremento paulatino en la oscilación térmica diaria que podría vulnerar la capacidad de respuesta del árbol (Ramírez et al., 2011).

En este caso, con la ausencia de lluvia durante el invierno de 2010-2011 se incrementó la oscilación entre las temperaturas mínimas y máximas diarias, lo que propició con mayor frecuencia la ocurrencia de temperaturas diarias mínimas por debajo de $0^{\circ} \mathrm{C}$ y máximas superiores a $15^{\circ} \mathrm{C}$; en tal circunstancia se acumula poco frío. Tabuenca 
(1975) había señalado previamente la baja acumulación de frío sólo por la ocurrencia de temperaturas altas durante el invierno en España.

La menor acumulación de UF durante el invierno coincidió con una reducción del $24 \%$ en la apertura de yemas en Royal Gala durante la primavera; en trabajos previos se reportó una situación similar con especies del género Ribes (Jones et al., 2013); además, la baja acumulación de frío en Cuauhtémoc, Chihuahua se asoció con un retraso de 90 GD para el inicio de floración en Royal Gala; en trabajos previos, se había señalado el retraso de la floración por baja acumulación de frío en duraznero, frambuesa (Rubus idaeus), grosella negra (Ribes nigrum) y pera (Pyrus communis) (Atkinson et al., 2013; Guédon y Legave, 2008; Legave et al., 2015).

Se ha reportado efecto variable del portainjerto en la floración de la variedad; en este trabajo el contraste entre portainjertos arrojó igualdad estadística en el requerimiento de calor para completar el evento de floración de Royal Gala; Tabuenca y Gracia (1971) así lo reportaron al evaluar 18 combinaciones generadas de tres portainjertos y seis variedades, mientras que Durner y Goffreda (1992) observaron efecto variable de portainjertos en la floración de duraznero Redhaven, Rio-Oso-Gem, Loring y Encore injertados sobre diferentes portainjertos. Por lo tanto, lo expuesto en los resultados es consistente con el retraso en el inicio de floración, pero difiere del señalamiento genérico de Atkinson et al. (2013) de que la baja acumulación de frío invernal propicia un alargamiento del periodo de brotación.

Posadas-Herrera et al. (2018) señalan que en Zacatlán, Puebla, con materiales que no son tardíos (precoces o intermedios) a la cosecha se evitan daños por heladas. En el oeste del estado de Chihuahua, con el retraso de la floración se reduce la probabilidad de daño por heladas tardías, pero se incrementa el riesgo de incidencia de mancha de fuego [Erwinia amylovora (Burril) Winslow] por la ocurrencia de temperaturas altas; de cualquier forma, en este ámbito en particular, se incrementan los costos de producción en inviernos con acumulación alta de frío por la aplicación de recursos económicos en la prevención de heladas y con baja acumulación de frío invernal por las actividades destinadas a la prevención de enfermedades y a la aplicación de estimulantes de brotación.

\section{CONCLUSIONES}

En el oeste del estado de Chihuahua, con la baja acumulación de frío invernal medido en unidades frío se redujo de manera significativa la apertura de yemas y

Cuadro 2. Valores medios del requerimiento de días grado (temperatura base de $4.5^{\circ} \mathrm{C}$ ) para inicio $(5 \%)$ y duración (de 5 a 95 \%) del evento de floración de manzano Royal Gala sobre diferentes portainjertos y frío invernal (bajo 524 UF; alto 830 UF) en Cuauhtémoc, Chihhuahua, México.

\begin{tabular}{|c|c|c|c|c|c|c|}
\hline \multirow{3}{*}{ Portainjerto } & \multicolumn{4}{|c|}{ Frío invernal (Unidades Frío) } & \multirow{2}{*}{\multicolumn{2}{|c|}{$\begin{array}{c}\text { Grados día por } \\
\text { Royal Gala/portainjerto }\end{array}$}} \\
\hline & Bajo & Alto & Bajo & Alto & & \\
\hline & \multicolumn{2}{|c|}{ Inicio } & \multicolumn{2}{|c|}{ Duración } & Inicio & Duración \\
\hline B.9 & 236 & 114 & 175 & 153 & $175 \pm 34 a$ & $135 \pm 18 a$ \\
\hline G.11 & 209 & 150 & 112 & 145 & $180 \pm 15 a$ & $129 \pm 14 a$ \\
\hline G.16 & 244 & 142 & 139 & 138 & $193 \pm 24 a$ & $139 \pm 7 a$ \\
\hline G.30 & 244 & 148 & 91 & 127 & $196 \pm 23 a$ & $109 \pm 12 a$ \\
\hline G.41 & 285 & 154 & 90 & 111 & $219 \pm 38 a$ & $100 \pm 16 a$ \\
\hline G.4210 & 235 & 186 & 124 & 84 & $211 \pm 16 a$ & $104 \pm 15 a$ \\
\hline G.6210 & 211 & 149 & 138 & 107 & $180 \pm 16 a$ & $123 \pm 14 a$ \\
\hline G.935 & 264 & 150 & 100 & 110 & $207 \pm 26 a$ & $105 \pm 14 a$ \\
\hline M.26 & 233 & 137 & 113 & 135 & $185 \pm 26 a$ & $124 \pm 10 a$ \\
\hline M.7 & 206 & 143 & 142 & 147 & $175 \pm 17 a$ & $144 \pm 6 a$ \\
\hline M.9PAJAM2 & 203 & 132 & 130 & 147 & $168 \pm 18 a$ & $139 \pm 9 a$ \\
\hline М.9Т337 & 257 & 150 & 110 & 146 & $203 \pm 26 a$ & $128 \pm 18 a$ \\
\hline $\begin{array}{l}\text { Días grado posterior a frío } \\
\text { invernal }\end{array}$ & $236 \pm 7 a$ & $146 \pm 5 b$ & $117 \pm 5 a$ & $129 \pm 6 a$ & & \\
\hline
\end{tabular}

Valores con misma letra en hilera frecuencia relativa por frío invernal significa igualdad estadística entre ellos, mientras que valores con misma letra en columnas de la derecha significa igualdad estadística entre portainjertos (Tukey, $\mathrm{P} \leq 0.05$ ). 
retrasó el inicio de floración (5 \%) en manzano Royal Gala, mientras que posterior a baja o alta acumulación de frío, la duración del periodo de floración en días grado fue estadísticamente igual. Con respecto a portainjertos, se detectó igualdad estadística para éstos en el porcentaje de apertura de yemas, inicio y duración del evento de floración. No se detectó interacción significativa entre frío invernal y portainjerto para las variables evaluadas.

\section{AGRADECIMIENTOS}

Se agradece a los revisores y editor técnico anónimos que tuvieron a bien revisar, sugerir y emitir comentarios para la mejora del escrito.

\section{BIBLIOGRAFÍA}

Allen B. C. and M. Wann (1983) Dormancy in apple tree buds. Biomathematics Series No. 13. Institute of Statistics Mimeo Series No. 1631. North Caroline State University. Raleigh, North Carolina, USA. 43 p.

Atkinson C. J., R. M. Brennan and H. G. Jones (2013) Declining chilling and its impact on temperate perennial crops. Environmental and Experimental Botany 91:48-62, https://doi.org/10.1016/j.envexpbot.2013.02.004

Denardi F., M. V. Kvitschal e M. C. Hawerroth (2015) Porta-enxertos de macieira: passado, presente e futuro. Agropecuária Catarinense, Florianópolis 28:89-95

Durner E. F. and J. C. Goffreda (1992) Rootstock-induced differences in flower bud phenology in peach. Journal of the American Society for Horticultural Sciences 117:690, https://doi.org/10.21273/JASHS.117.5.690

García E. (2004) Modificaciones al Sistema de Clasificación Climática de Köppen. Serie Libros No. 6. Instituto de Geografía, Universidad Nacional Autónoma de México. México, D.F. 90 p.

Guédon Y. and J. M. Legave (2008) Analyzing the time-course variation of apple and pear tree dates of flowering stages in the global warming context. Ecological Modelling 219:189199, https://doi.org/10.1016/j.ecolmodel.2008.08.010

Jacobo-Cuellar J. L., G. Mora-Aguilera, M. R. Ramírez-Legarreta, J. VeraGraziano, V. M. Pinto, J. López-Collado, M. E. Ramírez-Guzmán y L. A. Aceves-Navarro (2005) Caracterización cuantitativa de la diapausa de palomilla de la manzana Cydia pomonella L. en Cuauhtémoc, Chihuahua. México. Agrociencia 39:221-229.

Jones H. G., R. M. Hillis, S. L. Gordon and R. M. Brennan (2013) An approach to the determination of winter chill requirements for different Ribes cultivars. Plant Biology 15:18-27, https://doi.org/10.1111/j.1438-8677.2012.00590.x

Legave J. M., M. Blanke, D. Christen, D. Giovannini, V. Mathieu and R. Oger (2013) A comprehensive overview of the spatial and temporal variability of apple bud dormancy release and blooming phenology in Western Europe. International Journal of Biometeorology 57:317-331, https://doi.org/10.1007/s00484-012-0551-9

Legave J. M., Y. Guédon, G. Malagi, A. El Yaacoubi and M. Bonhomme (2015) Differentiated responses of apple tree floral phenology to global warming in contrasting climatic regions. Frontiers in Plant Sciences 6:1054, https://doi:.org/10.3389/fpls.2015.01054

Luedeling E. (2012) Climate change impacts on winter chill for temperate fruit and nut production: a review. Scientia Horticulturae 144:218-229, https://doi.org/10.1016/j.scienta.2012.07.011

Malagi G., M. R. Sachet, I. Citadin, F. G. Herter, M. Bonhomme, J. L. Regnard and J. M. Legave (2015) The comparison of dormancy dynamics in apple trees grown under temperate and mild winter climates imposes a renewal of classical approaches. Trees 29:13651380, https://doi.org/10.1007/s00468-015-1214-3

Marini R. P., B. Black, R. M. Crassweller, P. A. Domoto, C. Hampson, R. Moran, T. Robinson, M. Stasiak and D. Wolfe (2014) Performance of 'Golden Delicious' apple on 23 rootstocks at eight locations: a ten-year summary of the 2003 NC-140 dwarf rootstock trial. Journal of the American Pomological Society 68:54-68.

Mora-Aguilera G., D. Nieto-Angel, C. L. Campbell, D. Téliz and E. García (1996) Multivariate comparison of papaya ringspot epidemics Phytopathology 86:70-78, https://doi.org/10.1094/Phyto-86-70

Pennypacker S. P., H. D. Knoble, C. E. Antle and L. V. Madden (1980) A flexible model for studying plant disease progression. Phytopathology 70:232-235, https://doi.org/10.1094/Phyto-70-232

Posadas-Herrera B. M., P. A. López, N. Gutiérrez-Rangel, R. Díaz-Cervantes y A. Ibáñez-Martínez (2018) La diversidad fenotípica de manzano en Zacatlán, Puebla, México es amplia y es aportada principalmente por características de fruto. Revista Fitotecnia Mexicana 41:49-58.

Ramírez L. M. R., J. A. Ruiz C., G. Medina G., J. L. Jacobo C., R. A. Parra Q., M. R. Ávila M. y J. P. Amado Á. (2011) Perspectivas del sistema de producción de manzano en Chihuahua ante el cambio climático. Revista Mexicana de Ciencias Agrícolas 2:265-279.

Richardson E. A., S. D. Seeley and D. R. Walker (1974) A model for estimating the completion of rest for 'Redhaven' and 'Elberta' peach trees. HortScience 9:331-332.

Samish R. M. (1954) Dormancy in woody plants Annual Review of Plant Physiology 5:183-204, https://doi.org/10.1146/annurev.pp.05.060154.001151

SAS Institute (2011) SAS/STAT 9.3 User's Guide. SAS Institute Inc. Cary, North Carolina, USA. 8621 p.

Sincich T., D. M. Levine and D. Stephan (2002) Practical Statistical by Example Using Microsoft巴 Excel and Minitab. Second edition. Prentice Hall. Upper Saddle River, New Jersey, USA. 789 p.

Tabuenca M. C. (1975) Evaluación de las necesidades de frío para salir del reposo de variedades de almendro. Anales de la Estación Experimental de Aula Dei 13:208-217.

Tabuenca M. C. y M. Gracia (1971) Influencia del patrón de la época de salida del reposo invernal de la variedad. Anales de la Estación Experimental de Aula Dei 11:51-57.

UCARN, University of California Agriculture and Natural Resources (2019) Weather, models, \& degree-days. University of California Agriculture and Natural Resources, Statewide Integrated Pest Management Program. Mountain View, California, USA. http://ipm.ucanr.edu/WEATHER/index.html. (Septiembre, 2018).

Whiting M.D.,M. R.SalazarandG.Hoogenboom(2015)Developmentofbloom phenology models for tree fruits. Acta Horticulturae 1068:107112, https://doi.org/10.17660/ActaHortic.2015.1068.12 